\title{
USE OF THE VOICE-CONTROLLED AND COMPUTER-ASSISTED SURGICAL SYSTEM ZEUS FOR ENDOSCOPIC CORONARY ARTERY BYPASS GRAFTING
}

Hermann Reichenspurner, MD, PhD

Ralph J. Damiano, MD

Michael Mack, MD

Dieter H. Boehm, MD, PhD

Helmut Gulbins, MD

Christian Detter, MD

Bruno Meiser, MD

Reinhard Ellgass, MSc

Bruno Reichart, MD
Objective: With the aim of performing a completely endoscopic coronary bypass anastomosis, we have undertaken an experimental and clinical study using robotic instrumentation and voice-controlled camera guidance. Methods: The ZEUS Robotic Surgical System (Computer Motion Inc, Goleta, Calif) consists of three interactive robotic arms and a control unit, allowing the surgeon to move the instrument arms in a scaled down mode. The third arm (AESOP, Computer Motion) positions the endoscope via voice control. Phase I: In a phantom model, vascular grafts were anastomosed to the left anterior descending coronary artery (LAD) of 50 pig hearts with either 2- or 3-dimensional visualization. Phase II: In 6 dogs (FBI 20-25 kg) the left internal thoracic artery (LITA) was harvested endoscopically. Then the animals were placed on an endovascular cardiopulmonary bypass system (Port-Access, Heartport, Inc, Redwood City, Calif). Anastomosis of the LITA to the LAD was performed endoscopically with the telemetric ZEUS instruments. Flow rates through the LITA were measured by Doppler analysis. Phase III: Two patients were operated on with the ZEUS system. After endoscopic harvesting of the LITA and cardiopulmonary bypass with the Port-Access system, the bypass graft (LITA-LAD) was anastomosed endoscopically with the ZEUS system through three thoracic ports. Results: In the dry laborato$r y$, the time range required for the robotically assisted coronary anastomosis was 35 to 60 minutes with 2-dimensional visualization and 16 to 32 minutes with 3-dimensional visualization. In the animal experiments, the median time for endoscopic harvesting of the LITA was 86 minutes (range 56-120 minutes) and for the anastomosis, 42 minutes (range 35105 minutes); flow rates through the LITA ranged between 22 and 45 $\mathrm{mL} / \mathrm{min}$. In the clinical cases, preparation times for the LITA were 83 and 110 minutes, respectively, and anastomosis times, 42 and 40 minutes, respectively. Doppler flow rates measured 125 and $85 \mathrm{~mL} / \mathrm{min}$, respectively. Both patients had an uneventful follow-up angiogram and postoperative course. Conclusions: With sophisticated robotic technology, a completely endoscopic anastomosis of the LITA to the LAD is possible, allowing technically precise operations within acceptable time limits. (J Thorac Cardiovasc Surg 1999;118:11-6)
S everal different techniques have been established for the less invasive surgical treatment of coronary artery disease. However, small incisions, such as

From the Department of Cardiac Surgery, University Hospital Munich-Grosshadern, D-81366 Munich, Germany; Division of Cardiothoracic Surgery, Milton S. Hershey Medical Center, Pennsylvania State University, Hershey, Pa; and Dallas Medical Center, Dallas, Tex

Received for publication Dec 1, 1998; revisions requested Jan 21, 1999; revisions received March 22, 1999; accepted for publication March 23, 1999. minithoracotomy or partial sternotomy, are still necessary to do the coronary artery anastomosis procedure. The performance of a manual endoscopic coronary

\footnotetext{
Address for reprints: Hermann Reichenspurner MD, PhD, Department of Cardiac Surgery, University Hospital Grosshadern, Marchioninistr 15, D-81377 Munich, Germany.
}

Copyright ( 1999 by Mosby, Inc.

$0022-5223 / 99 \$ 8.00+0 \quad \mathbf{1 2 / 1 / 9 8 7 2 8}$ 


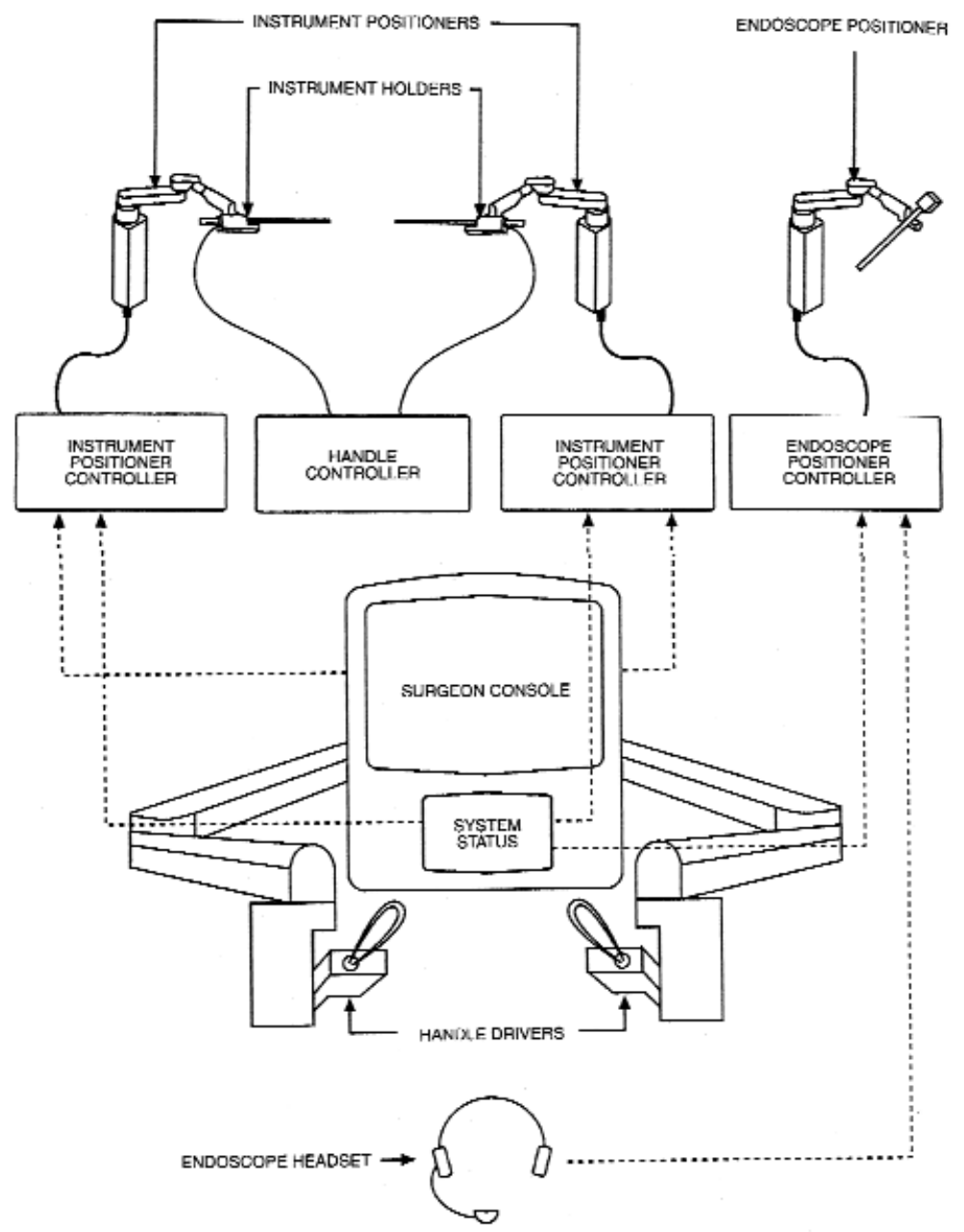

Fig 1. Schematic drawing of the ZEUS system consisting of the surgeon console and 3 interactive robotic arms mounted onto the operating table (for detailed explanation, see text).

artery bypass grafting procedure has not been achieved yet and would be extremely difficult and limited to few surgeons only. For this reason, computer-assisted systems have been developed to assist the surgeon in endoscopic coronary artery surgical procedures. A specifically designed system has been used at the Hôpital Broussais (Loulmet D, Carpentier A. Personal communication, 1998) and the University of Leipzig ${ }^{1}$ for operations in patients with mitral valve disease and coronary artery disease. This is a description of the experimental and first clinical use of the voice-controlled and computer-assisted ZEUS Robotic Surgical System (Computer Motion Inc, Goleta, Calif). The ZEUS system was designed to duplicate the degrees of freedom (DOF) obtained with the use of a standard endoscopic instrument controlled by the hand of the surgeon. In precise terms, the DOF with the ZEUS sys- tem include vertical, horizontal, in and out, rotational, and grasping movements that equate to 5 DOF inside the chest. In addition, natural tremor is filtered completely and the instrument movements are scaled to improve the surgeon's dexterity and ergonomics.

In both clinical cases the coronary artery bypass anastomoses were done through endoscopic ports only.

\section{Material and methods}

The ZEUS Robotic Surgical System consists of three interactive robotic arms placed at the operating table, a computer controller, and an ergonomically enhanced surgeon console (Fig 1). One robotic arm is used to position the endoscope (AESOP, Computer Motion Inc.), and the other two robotic arms manipulate surgical instruments under the surgeon's direct control. While seated at the console in a chair with armrests, the surgeon can have either a 3-dimensional or a 2dimensional view of the operative site, depending on prefer- 


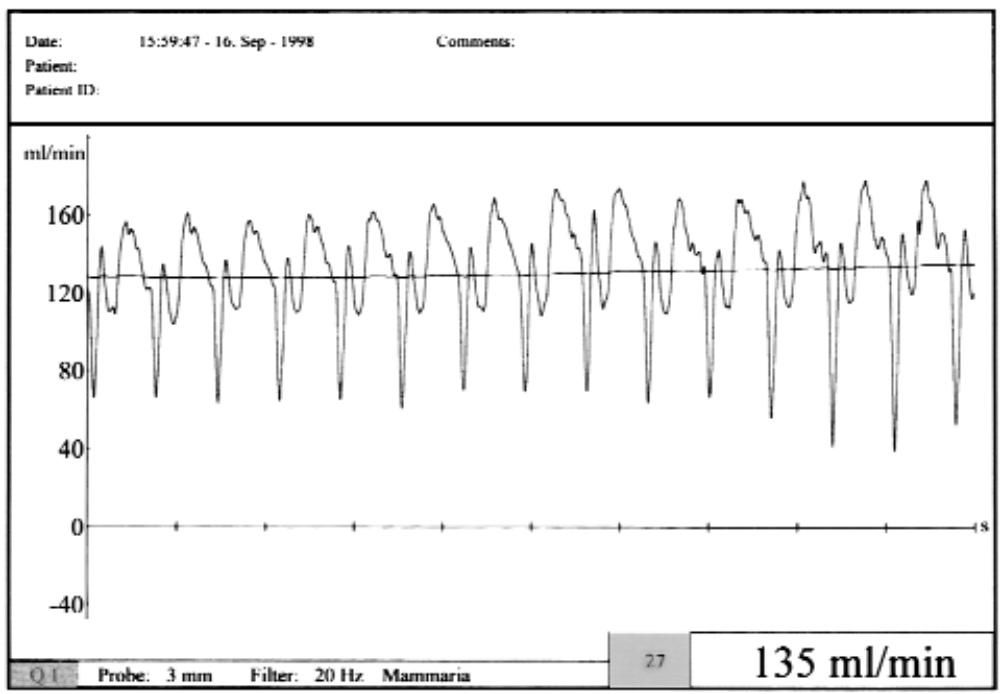

Fig 2. Intraoperative Doppler flow analysis demonstrating a LITA flow of $135 \mathrm{~mL} / \mathrm{min}$.

ence. The surgeon controls the movements of the endoscope with simple spoken commands. Movements of the surgical instruments are controlled via handles that resemble conventional surgical instruments. The movements of the instrument handles are scaled and tremor is filtered such that the surgeon is able to perform fully endoscopic and accurate microsurgery.

The instrument positioner controllers form the link between the surgeon at the surgeon console and the instruments. These controllers analyze the input data measured at the surgeon console as the surgeon moves the handles and then direct the instrument positioners to move appropriately so that the movements of the instrument tips directly replicate that of the surgeon at the console. The computer is also able to scale the input data because the physical link between surgeon and instrument has been replaced with a digital one (Fig 1).

Visual control was obtained with either 3-dimensional (Zeiss $\mathrm{GmbH}$, Oberkochen, Germany) or 2-dimensional (Karl Storz $\mathrm{GmbH}$, Tuttlingen, Germany) endoscopes. The 3-dimensional image was displayed on two monitors inside a special headset (Vista Technologies Inc, Westborough, Mass) and a 2-dimensional image via a monitor above the control unit.

I. Phantom training. During the first phase, the system was tested in a phantom model, in which simple motions and knot-tying techniques were evaluated. Subsequently, end-toside anastomoses were performed with porcine thoracic or excised coronary arteries to the left anterior descending coronary artery (LAD) of 50 explanted pig hearts that were placed in an anatomic position inside the phantom model.

II. Animal experiments. In 6 dogs (FBI, 20-25 kg), the left internal thoracic artery (LITA) was harvested endoscopically via a $10-\mathrm{mm}$ camera port and two 5-mm instrument ports. The 3-dimensional endoscope was used and the surgeon was wearing the headset with a 3-dimensional display of the endoscopic image. Camera movement was voice-con- trolled with the AESOP robotic arm. After the distal LITA had been clipped, the animals were placed on cardiopulmonary bypass (CPB) by femoral cannulation with the use of the Port-Access system (Heartport Inc, Redwood City, Calif).

After endoscopic clamping of the LITA, the distal end of the LITA was pulled through one of the thoracoscopic ports to prepare the distal end for anastomosis.

After insertion and inflation of the endoaortic balloon catheter (Endoclamp, Heartport) using fluoroscopic control and antegrade delivery of cold crystalloid cardioplegic solution, the pericardium was opened with the aid of the ZEUS system, which was inserted through the same three left lateral endoscopic ports. The LAD was dissected with specially designed beaver knives, which were attached to the ZEUS instrument driver. The LAD was then incised with the same instruments.

The end-to-side-anastomosis (LITA-LAD) was performed with the aid of the ZEUS system with 7-0 polytetrafluoroethylene sutures* through the three lateral ports in 5 cases and through three subxiphoid ports in 1 case. The operation was assisted through a right parasternal thoracoscopic port, through which the assistant passed an endoscopic forceps. After completion of the anastomosis the endoscopic bulldog clamp was removed and the bypass graft was perfused. To assess flow rates, the proximal portion of the LAD was ligated with a 4-0 Prolene suture (Ethicon, Inc, Somerville, NJ) with the aid of the ZEUS system. At the end of the procedure, blood flow through the LITA graft was assessed by Doppler transit-time flow measurement after the animals were weaned from CPB. The transit-time principle measures the blood flow directly without needing additional information regard-

*Gore-Tex sutures; registered trademark of W. L. Gore \& Associates, Inc, Flagstaff, Ariz. 


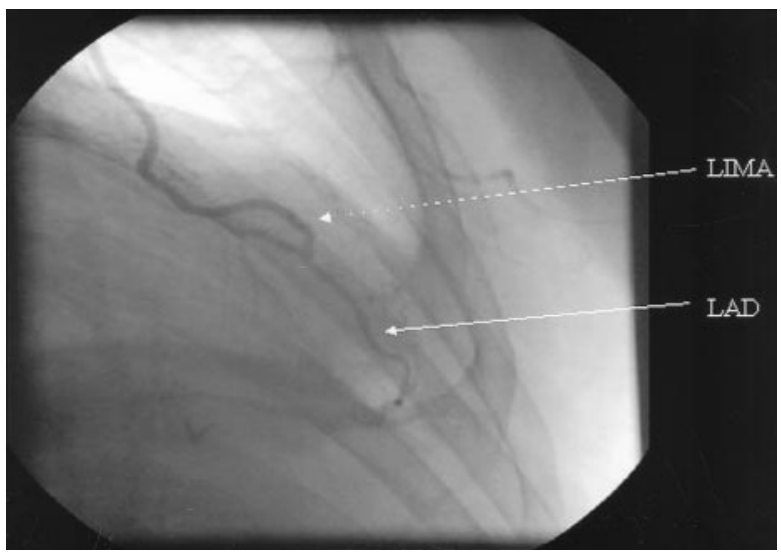

Fig 3. Postoperative angiogram showing a patent and uneventful anastomosis of the LITA to the LAD.

ing the angle of the incident ultrasound wave or vessel diameter. An appropriately seized probe is positioned around the vessel. The principle of measurement is based on the fact that the time required for ultrasound to pass through blood is slightly longer when it is passing upstream than downstream. The two ultrasound crystals are placed on one side of the probe and a reflector on the opposite side. Ultrasound is transmitted from the first crystal downstream and received at the second crystal after a time delay (t1). After reversal of the function of the two crystals, the transit time ( 2 ) across the blood flow is measured. The resulting transit time difference $\mathrm{t} 2-\mathrm{t} 1$ is proportional to the true blood volume flow.

At subsequent autopsy, the anastomotic sites were checked for patency.

III. Clinical cases. After approval by the Ethics Committee of the University of Munich and written informed consent were obtained, two patients with proximal stenosis of the LAD were selected for the clinical procedures. Endoscopic LITA harvesting was done first through three left lateral thoracic ports penetrating the fourth, fifth, and sixth intercostal space. The 10-mm camera port was inserted at the level of the anterior axillary line and the two instrument ports to the left and right of the camera port at the level of the midaxillary line. The LITA was dissected manually by means of electrocautery and endoscopic clipping of side branches under carbon dioxide inflation. Before distal clipping of the LITA, a left parasternal minithoracotomy was done through the fourth intercostal space to enable one of the surgeons to assist in the case and assess the safety of instrument placement. The LITA was then clipped distally, pulled through the minithoracotomy, and prepared for the anastomosis.

In parallel, the right femoral artery and vein were dissected and surrounded with umbilical tapes. The specifically designed Port-Access femoral cannulas were inserted into the femoral vessels. The venous cannula was guided into the right atrium with the aid of transesophageal echocardiographic control. After placement of the arterial cannula, the Endoclamp was advanced into the ascending aorta with fluoro- scopic and transesophageal echocardiographic control. CPB was then established, and the heart was emptied. Additional drainage was achieved through the previously placed Endopulmonary vent catheter (Heartport).

In the first case, the endoscopic coronary artery anastomosis was performed through the same left thoracic ports that were used for LITA takedown. The 3-dimensional endoscope was reinserted through the camera port and the instrument drivers were inserted through the two instrument ports.

Through the minithoracotomy, the pericardium was opened and the LAD was dissected. After preparation of the whole situs, the endoaortic balloon was inflated until aortic occlusion was achieved and antegrade blood cardioplegia was administered for cardiac arrest. The position of the balloon was monitored initially with fluoroscopy and consecutively with transesophageal echocardiography. After completed application of cardioplegia, the proximal aortic vent was connected. The LAD was incised through the minithoracotomy and the LITA was placed next to the LAD. A 7-0 polytetrafluoroethylene suture was placed into the needle holder of the system and the end-to-side anastomosis was performed with a running suture through the three endoscopic ports only.

In the second patient, the camera and instrument ports for the coronary anastomosis were placed in a subxiphoid position with the left instrument port being placed directly under the xiphoid and the camera and instrument ports being placed $3 \mathrm{~cm}$ to the right of the neighboring port. The coronary artery anastomosis was then done endoscopically through these three subxiphoid endoscopic ports.

At the end of the anastomosis, the suture was tied seven times with the same instruments. The endoscopic bulldog clamp was removed and the anastomoses were tested for hemostasis. No additional suture placement was necessary. Thereafter, the endoaortic balloon was deflated and the heart was perfused. In both cases the heart defibrillated spontaneously. After 20 minutes of reperfusion, the patients were weaned from $\mathrm{CPB}$ without catecholamine support. Bypass flow rates were analyzed with Doppler flow probes. Intraoperative angiography was performed through the Yshaped arterial cannula by the cardiologist. After decannulation, protamine was administered and the chest was closed after insertion of one chest drain.

Total time of the surgical procedure, time of CPB, crossclamp time, time of ventilatory support, time in the intensive care unit, and total hospital stay were recorded.

\section{Results}

Simulator training. Different imaging techniques for coronary anastomoses were evaluated by three surgeons using either 2-dimensional or 3-dimensional visualization. The median time of coronary anastomoses with the 2-dimensional view was 42 minutes (range 35-60 minutes) and with the 3-dimensional view, 20 minutes (range 12-32 minutes) $(P<.05,2$ dimensional vs 3-dimensional).

Experimental data. The median time for LITA har- 
vesting in the animal experiments was 86 minutes (range 56-120 minutes). In all six cases, it was possible to harvest the LITA endoscopically. The animals were then placed on Port-Access CPB. Median time of CPB was 95 minutes (range 82-200 minutes). Median time of aortic occlusion was 65 minutes (range 53-125 minutes) and for the anastomosis, 42 minutes (range 35105 minutes). All animals but one were weaned from CPB without major catecholamine support. One animal showed signs of impaired myocardial protection during the procedure. Despite an acceptable LITA flow rate, the animal was not able to be weaned from CPB. The median LITA flow rate, measured by Doppler flow assessment, was $38.5 \mathrm{~mL} / \mathrm{min}$ (range $22-45 \mathrm{~mL} / \mathrm{min}$ ). At autopsy, all explanted anastomoses showed good patency with no evidence of stenoses.

Clinical cases. In the patients, the times for endoscopic LITA preparation were 83 minutes and 110 minutes, respectively. In the first patient, bleeding occurred from one of the LITA side branches and was finally stopped through the minithoracotomy. The setup of the ZEUS instruments was done in parallel to the installation of the Port-Access CPB. The times for ZEUS system setup were 17 and 21 minutes in the two patients. Total CPB times were 62 and 95 minutes. The aorta was occluded for 55 and 52 minutes. The exact times for the coronary anastomoses were 42 and 40 minutes, respectively. Both patients were weaned from CPB without major catecholamine support. Subsequently, the Doppler flow rates of the bypass grafts were measured to be 135 and $85 \mathrm{~mL} / \mathrm{min}$, respectively (Fig 2). Intraoperative angiographic studies were performed in both patients and showed patent anastomoses without evidence of narrowing or stenoses.

Total time of the operation was 4.5 hours in the first patient and 5.3 hours in the second patient.

Postoperative time in the intensive care unit was 8 hours and 2 days; the second patient had a transient postoperative delirium that resolved after 48 hours. Total hospitalization was 5 days and 8 days, respectively, and both patients were discharged to their homes in excellent condition. Postoperative follow-up at 6 weeks is complete in both patients, with no evidence of wound infection, wound pain, or recurrent angina pectoris. Electrocardiograms, chest x-ray films, and follow-up angiograms were uneventful (Fig 3).

\section{Comment}

This report presents experimental data and the first successful clinical use of the computer-assisted and voice-controlled ZEUS system in performing endoscopic coronary artery bypass anastomoses. The preclinical experiments proved that 3-dimensional visualization enabled a significant reduction in the time required for a coronary bypass anastomosis. Three-dimensional visualization leads to more realistic depth perception and improves surgical dexterity; it therefore involves a shorter learning curve and faster anastomosis times.

Within the animal experiments, as well as in both clinical cases, a successful endoscopic coronary artery anastomosis was performed with the ZEUS system without any complications, such as bleeding or anastomotic stenosis; these findings have been confirmed in previous animal studies in which the same system was used. ${ }^{2}$ For safety reasons, small chest incisions were made in both patients to give the assistant the possibility to intervene immediately in case of any complication. The times of anastomoses were still longer than those needed for regular coronary artery anastomoses; however, with more experience, particularly in clinical situations, the anastomosis times can be expected to approximate those achieved in regular coronary artery surgery. The total operative and crossclamp times were comparable with our own initial experience with PortAccess coronary artery bypass surgery. ${ }^{3}$ Patient safety was guaranteed by the additional minithoracotomy, which allowed the assistant total control of the operative field.

The advantage of the ZEUS surgical systems was the ability to set up the system quickly, within 15 minutes. The robotic arms can be attached to the table before the operation, without interfering with endoscopic LITA harvest, initiation of Port-Access CPB, the assistance by the second surgeon, and the instrumentation by the surgical nurse. The system was simple to handle and did not exhibit any technical failures during the experiments or the clinical cases. Other devices have as many as 7 DOF. The ability to add DOF has also increased the size of the instrumentation. For example, the instruments that support the 7-DOF system are 11 $\mathrm{mm}$ in diameter, contrasted with the $3.9-\mathrm{mm}$ size of the ZEUS instruments. The ability of the ZEUS system to perform the delicate motions required to complete the cardiac anastomosis, dissect, grasp, cut, and manipulate tissue is well documented in both clinical procedures and animal experiments. ${ }^{2}$ In addition to those clinical cardiac surgical procedures, the ZEUS surgical system has been used in experimental and clinical cases of tubal reanastomoses in gynecology. ${ }^{4}$

As important as the DOF provided by the device is the operating room compatibility. Operating room compatibility can be defined as patient safety, work space ergonomics, flexibility of setup, rapid instrument changing, and overall footprint of the device. These factors contribute significantly to the ultimate clinical utility of a robotic surgical system. The competitive 
device with 7 DOF has been used in coronary artery operations at the Universities of Paris and Leipzig. In these cases the system has been used for endoscopic thoracic artery dissection and/or coronary artery bypass anastomosis. Total time of surgery, Port-Access CPB time, and aortic crossclamp time were comparable with those of the cases described herein. These cases include two fully endoscopic cases performed at Hôpital Broussais (Loulmet D, Carpentier A. Personal communication, 1998). ${ }^{1}$

Adequate training time is essential to use the described methods in conjunction with 3-dimensional technology if a technically precise operation is to be done within acceptable time limits. The final goal of endoscopic coronary artery surgery requires a stepwise approach including adequate training. Endoscopic LITA preparation, the Port-Access CPB system, a 3- dimensional visualization system, and a voice-controlled and computer-assisted surgical robotic system are ideal preconditions to perform truly endoscopic coronary artery bypass procedures.

\section{REFERENCES}

1. Mohr FW, Falk V, Diegeler A, Autschbach R: Computerenhanced coronary artery bypass surgery. J Thorac Cardiovasc Surg 1998;117:1212-5.

2. Stephenson ER, Sankholkar S, Ducko CT, Damiano RJ. Robotically-assisted microsurgery for endoscopic coronary artery bypass grafting. Ann Thorac Surg 1998;66:1064-7.

3. Reichenspurner H, Gulielmos V, Wunderlich J, Dangel M, Wagner FM, Pompili MF, et al. Port-Access coronary artery bypass grafting with the use of cardiopulmonary bypass and cardioplegic arrest. Ann Thorac Surg 1998;65:413-9.

4. Margossian H, Garcia-Ruiz A, Falcone T, Goldberg J, Attaran M, Gagner M. Robotically assisted laparoscopic microsurgical uterine horn anastomosis. Fertil Steril 1998;70:530-4.

\section{Authoritative}

The Journal of Thoracic and Cardiovascular Surgery is the most frequently cited thoracic/cardiovascular surgery journal in the Science Citation Index. An article in JTCVS is sited on average almost twice as often as those in the closest cardiothoracic journal. 